\title{
Preface
}

It is interesting thus to follow the intellectual truths of analysis in the phenomena of nature. This correspondence, of which the system of the world will offer us numerous examples, makes one of the greatest charms attached to mathematical speculations.

PieRRE-SimON LAPLACE

(1749-1827)

Mathematical discoveries, small or great are never born of spontaneous generation. They always presuppose a soil seeded with preliminary knowledge and well prepared by labour, both conscious and subconscious.

HENRI PoINCARE (1854-1912)

This book on the theory and simulation of random differential equations came into being as the result of our lecture "Dynamical Systems \& Scientific Computing - Introduction to the Theory $\&$ Simulation of Random Differential Equations" during the summer term 2012. This novel interdisciplinary way to cover dynamical systems and scientific computing brought Master students into contact with cutting edge research and was awarded the Ernst-Otto Fischer prize for innovative and trend-setting teaching paradigms by the department of Computer Science of the Technische Universität München.

Figure 1 provides a short overview of the pieces of this lecture and their fit. We will discuss this course and in particular the workshop on random differential equations and their application to ground motion excited multistorey buildings in chapters 17 and 18. These hands-on lecture notes serve as the theoretical foundation for our lecture and the workshop. MATLAB com- 
mands ${ }^{1}$ blend theory with application, and provide a solid foundation of the principles of deterministic ordinary differential equations and their numerics.

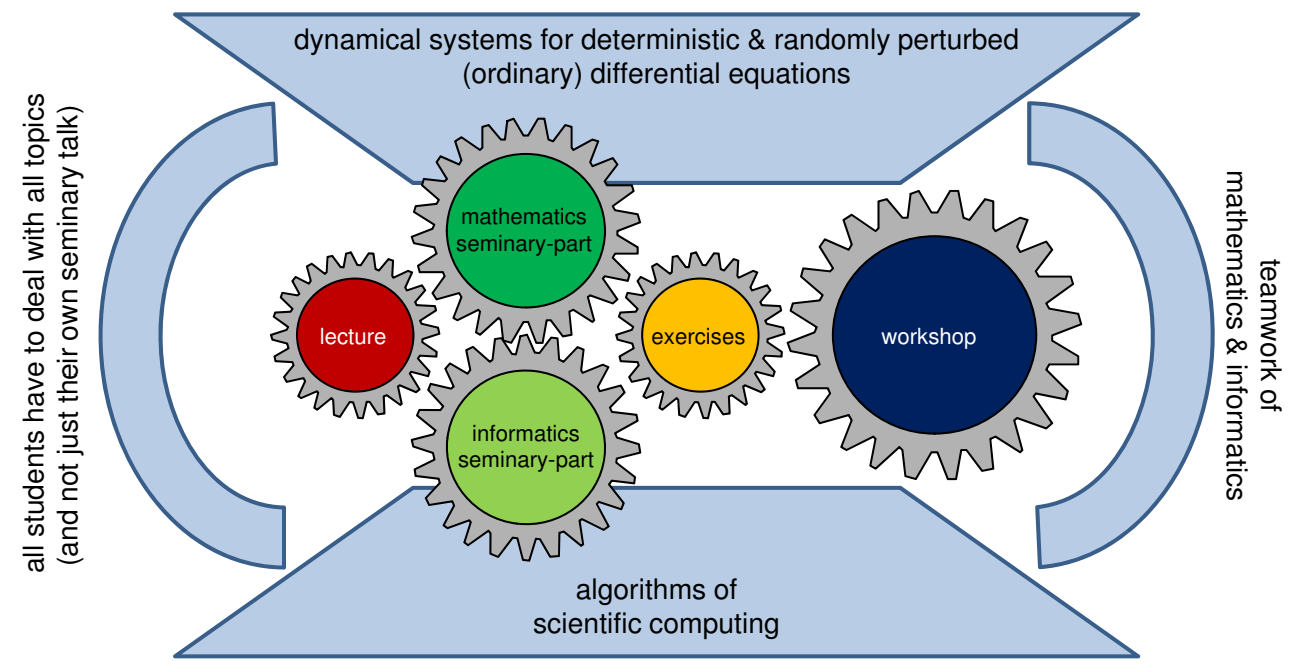

Figure 1. Design of the lecture "Dynamical Systems \& Scientific Computing - Introduction to the Theory \& Simulation of Random Differential Equations" combining the workshop with seminar contributions, exercises, and few lectures to increase student-centred learning effects.

Concerning random differential equations there is just a limited amount of literature discussing them on a graduate level, and despite their significance in scientific and engineering applications there is only one book in German by Helga Bunke [45] from 1972 and another one in Polish by Dobieslaw Bobrowski [30] from 1987 totally dedicated to the theory of random differential equations. T. T. Soong's monograph [233] from 1973 dedicates about half of his work to these equations. Arnold Kistner's PhD thesis [157] from 1978 (in German, too) covers essential aspects of linear random (ordinary) differential equations. There are a couple of recent papers and the one or other book chapter on the numerics of random differential equations. However, a holistic approach is missing in particular taking into account recent results of Ludwig Arnold on random dynamical systems, cf. [11].

This book is a holistic and self-contained treatment of the analysis and numerics of random differential equations from a problem-centred point of

Note that the MATLAB examples presented throughout this book are mainly meant to illustrate certain individual aspects in a compact manner; hence, these examples do not represent a "nice" implementation from a software engineering point of view (mostly, we skipped documenting comments for the sake of a compact representation, e.g.). 
view. We take an interdisciplinary approach by considering state-of-the-art concepts of both dynamical systems and scientific computing. Our intended audiences are those of beginning graduate/ master level courses on theory and numerics of stochastically perturbed differential equations. The areas covered here are of importance for interdisciplinary courses in informatics, engineering and mathematics. Increasing interest in "Uncertainty Quantification" during recent years warrants a textbook that is aimed at a new generation of researchers in this field, and which is rooted in the principles of dynamical systems and scientific computing. This will foster a solid understanding of both theory and simulation.

From a methodological point of view, the red line pervading this book is the two-fold reduction of a random partial differential equation disturbed by some external force as present in many important applications in science and engineering. First, the random partial differential equation is reduced to a set of random ordinary differential equations in the spirit of the method of lines. These are then further reduced to a family of (deterministic) ordinary differential equations, see Fig. 2 . In particular, this latter reduction step and the fields of mathematics and computer science which support it, form the basis of this exposition.

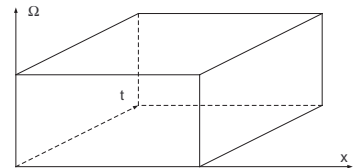

Space-Time-Realization-Cube

Partial Differential Equation

with Stochastic Effects (RPDE or SPDE)

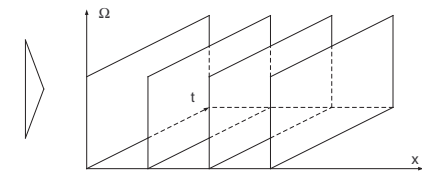

Space-Discretization (Finite Differences) Finite-Dimensional System of $\mathrm{R}(\mathrm{O}) \mathrm{DE}$

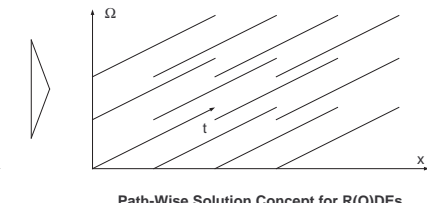

Path-Wise Solution Concept for R(O)DEs Finite-Dimensional System of an Infinite
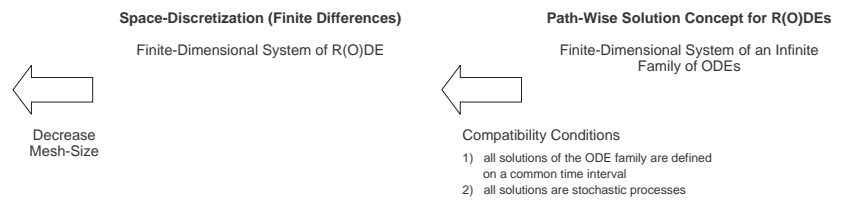

Figure 2. Reduction from a given continuum mechanical random partial differential equation to a family of deterministic ordinary differential equations.

Hereby, our main example is the motion of multi-storey buildings subject to stochastic ground motion excitations. The (simplified) buildings are either modeled as solids by standard assumptions of continuum mechanics (and their corresponding partial differential equation description) or wireframestructures based on deterministic oscillators. The external forcing is due to a linear filtered white noise that describes the earth's surface as a layer between the ground surface and the nearest bedrock where the source of an earthquake is located and treats the wave propagation in this layer as being one-dimensional and vertical. The corresponding stochastic models are known as the Kanai-Tajimi filter or the Clough-Penzien filter. 
From a didactical point of view, we put much effort into providing the deterministic foundation of the theory and simulation of ordinary differential equations as well as that of random variables and stochastic processes in order to give a self-contained presentation. Every chapter begins with a list of key concepts and key questions the reader should keep in mind while studying the contents of the respective chapter. Moreover, quite uniquely for a mathematics text book, every sub-chapter ends with a set of quizzes in the type of oral exam questions, allowing the knowledge obtained to be consolidated quickly, and to enable a successful self-study of the materials covered.

\section{Outline of the Chapters}

Figure 3 sketches the rough outline of this book and focuses on randomly perturbed phenomena in science and engineering, their mathematical analysis and effective as well as efficient numerical simulation. In contrast to the "classical" bottom-up textbook approach, we follow an application oriented top-down procedure, and proceed from discussions of complex applications to simpler known concepts for the following reason: This allows us to start with the complete picture and introduce the reader to applications, numerics and general theory quickly. Thus, in part I, we proceed from random partial differential equations (RPDEs), to random ordinary differential equations (RODEs) and then finally to ordinary differential equations (ODEs). During the lecture on which the book is based, we saw that the students struggled with the new concept of randomized ODEs at first and actually required knowledge on stochastic ordinary differential equations (SODEs) and other solution concepts to fully place RODEs into their body of knowledge and to fully appreciate the stochastic concepts. After the discussion of the "complete picture" we continue, in parts II and III, with a recap in the classical way, because of the interdisciplinary background of the intended readership, we believe that this is necessary in order to give a self contained representation. In particular, the chapters are such that they may be skipped by those readers familiar with the corresponding concepts. The main part of the exposition proceeds in part IV with a discussion of those RODEs that can be treated more or less easily: the linear ones. Here, the existence and uniqueness results are based on the general theorems provided in part I. Finally, RODEs and simulations together with their evaluation are joined in the workshop part $\mathrm{V}$.

In particular, the single chapters of this book contain the following specific information:

Part I serves as an introduction to the modelling of randomly perturbed phenomena in science and engineering by random partial differential equations and their reduction to random ordinary differential equations. Here, we discuss the following aspects: 


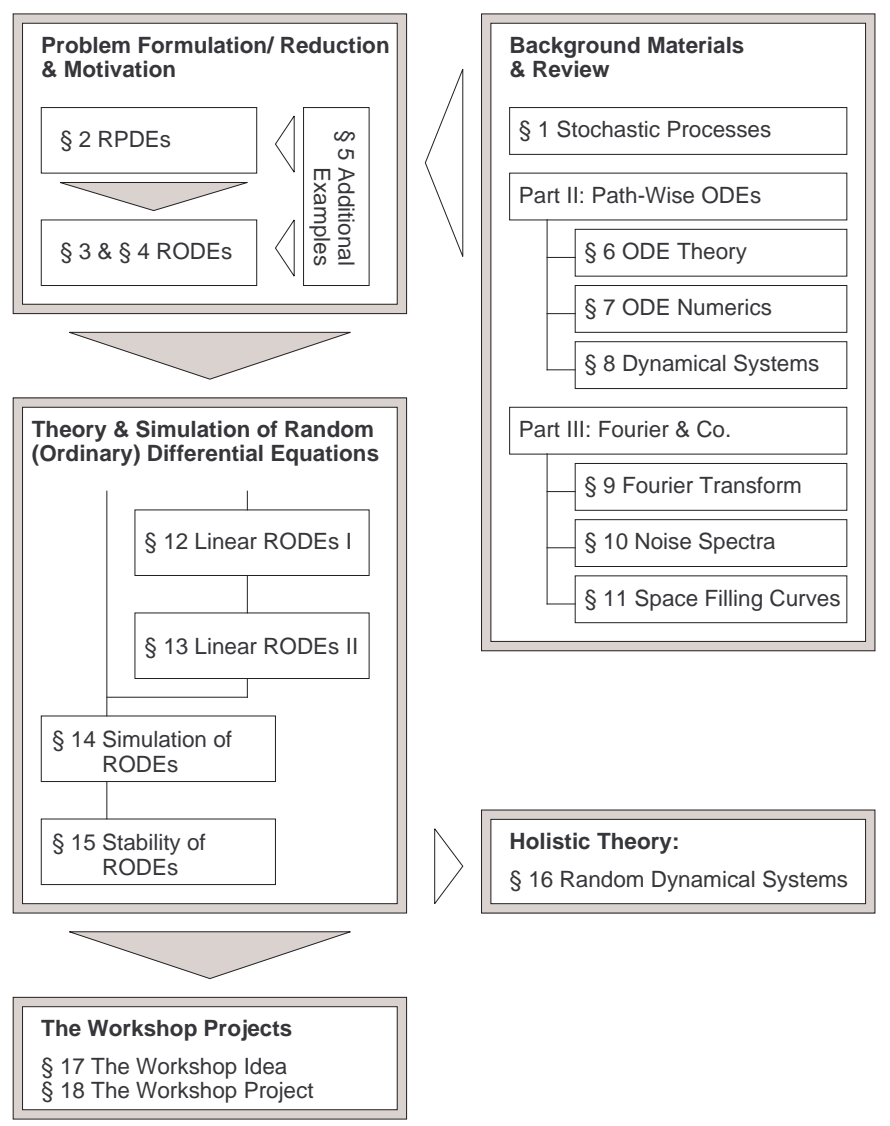

Figure 3. Outline of the book from the point of view of theory and simulation of random (partial/ ordinary) differential equations.

Chapter 1 provides a friendly review of the central concepts of probability theory focusing on random variables and their properties that eventually lead to the notion of a stochastic process. Our aim is to recall the basic definitions and equip them with tailored illustrations and MATLAB commands rather than emphasize the most general and abstract mathematical concepts.

Chapter 2 discusses how specific Random Partial Differential Equations are transformed to Random Ordinary Differential Equations by applying classical spatial discretisations. Variants from a variety of applications, are discussed, leaving the time discretisation for Chap. 7. The derivation of the underlying system of (deterministic) partial differential equations and corresponding boundary conditions is shown for the example of 
elastic body motion. We discuss different types of meshes with an emphasis on regular Cartesian grids. The three main spatial discretisations-finite differences, finite volumes, and finite elements-are briefly explained before delving deeper into finite difference schemes (FD). We derive the corresponding FD approximations for the fundamental equations of elastic body motion and simulate steady-state scenarios of buildings which are bent.

Chapter 3 motivates and mathematically rigorously discusses existence and uniqueness of path-wise solutions of random (ordinary) differential equations. We start by modelling external and ground motion excitations by means of stochastic processes which motivates the study of random (ordinary) differential equations. Their solution, existence and uniqueness concepts are then discussed together with the correspondence between stochastic and random (ordinary) differential equations. In particular, we study the conditions that lead to the existence of pathwise unique solutions. Solutions in the extended sense are analysed as well as the dependence of solutions on parameters and initial conditions. As an excursion we finally give the equations of motion for single- and multi-storey (wireframe) buildings. Our main source for the set-up and discussion of random differential equations is Helga Bunke's book [45].

Chapter 4 adds the notions of $\mathbb{P}$ - and mean-square solutions to our discussion. The special nature of random (ordinary) differential equations often requires additional refined solution concepts going beyond that of a path-wise solution. Taking, for instance, into account that a solution may fulfill the given random differential equation with probability one, or that the solution is a square integrable stochastic process leads to the notion of a $\mathbb{P}$-solution or a mean-square solution, respectively. Their properties and interconnections, in particular with respect to path-wise solutions, are studied here.

Chapter 5 widens the scope to additional categories of applications for random differential equations. In particular, flow problems are discussed in more detail. These problems represent an important class of applications in computational science and engineering. The various possible random effects in the model, the geometry, the boundary conditions, and the parameters may be generalised to other flow scenarios, involving coupled scenarios such as fluid-structure interaction or biofilm growth.

Part II is a mini-course on the interplay between dynamical systems and scientific computing in itself. Here, we cover the analytical and numerical foundations of deterministic ordinary differential equations. A special emphasis is given to dynamical systems theory including essential phase space 
structures (equilibria, periodic orbits, invariant sets) as well as fundamental tools (Lyapunov exponents and Lyapunov functions).

Chapter 6 serves as a holistic introduction to the theory of ordinary differential equations (without singularities). After some preliminaries, integral curves in vector fields are discussed, i.e., ordinary differential equations $\dot{x}=F(t, x)$. Hereby, we start with continuous right hand sides $F$ and their $\varepsilon$-approximate solutions as well as the Peano-Cauchy existence theorem and its implications. We continue our discussion for Lipschitzcontinuous functions $F$ and the existence and uniqueness theorem of Picard-Lindelöf. In particular, we analyse maximal integral curves, give the three types of maximal integral curves that can occur in autonomous systems and show the transformation of a $d$-th order equation into a first order system. Next, we deal with the existence of solutions in the extended sense where the right hand side function may be continuous except for a set of Lebesgue-measure zero. Caratheodory's existence theorem and its implications are studied together with maximum and minimum solutions. Then, we study the broad class of linear ordinary differential equations by discussing the unique existence of their solutions and their explicit construction. Applications of the theory focus on first integrals and oscillations for the deterministic pendulum and the Volterra-Lotka system. Finally, we provide a first glance into the existence, uniqueness and extension of solutions of ordinary differential equations on infinite-dimensional Banach spaces.

Chapter 7 contains the relevant aspects of the numerical simulation of ordinary differential equations. Classical explicit one-step methods such as the explicit Euler or Runge-Kutta schemes are presented before motivating implicit approaches for stiff ODEs. A variety of example implementations show the behaviour of the different schemes applied to different initial value problems. The brief discussion of the Newmark family of schemes and of symplectic methods widens the scope of this chapter to approaches that are typically neglected but that provide useful features worth being on the radar in the context of RODE simulations.

Chapter 8 provides a brief review on deterministic dynamical systems. Fundamental notions and concepts are introduced, like that of (continuous) dynamical systems, long-time behavior, invariance and attraction. This paves the way to analyze stability in the sense of Lyapunov by utilizing Lyapunov-functions for proving (asymptotic) stability in non-linear systems. Next, we analyze the correspondence between the stability properties of non-linear systems and their linearisation. Here, we give the famous theorem of Hartman and Grobman, a classification of equilibria in planar systems with respect to their stability properties as well as 
the techniques for the determination of the position of Lyapunov exponents of a linear system, like the Routh-Hurwitz criterion or the Lozinskiimeasure method.

Part III covers important concepts and algorithms in Scientific Computing: the discrete Fourier transform and its variants, the frequency domain method for response analysis, as well as space-filling curves as paradigms for effective and efficient data storage.

Chapter 9 discusses the basic aspects of the continuous and the discrete Fourier transform, with the focus on the latter including various MATLAB examples. The famous Fast Fourier Transform is derived. We briefly present the trigonometric variants of the discrete Fourier transform related to symmetry properties of the underlying input data. These trigonometric transforms allow us to realise fast Poisson solvers on Cartesian grids which are needed in the workshop problem (cf. Chap. 18). Frequency domain aspects and the Fourier transform are essential to understand key characteristics of stochastic processes (spectrum, power spectrum) and the propagation of excitations through mechanical structures.

Chapter 10 starts with the basic definitions and implications related to the spectral representation of stationary and periodic stochastic processes. Based on these, we study the notions of energy, power and spectral density. We give several examples for colored noise processes, the frequency domain method for response analysis, and linear filters. In particular, we apply this method to our problem of multi-storey excitation due to seismic impacts and their propagation through wireframe structures.

Chapter 11 introduces the fundamental concepts, definitions, and properties of space-filling curves such as the Hilbert and Peano curves. We briefly present three different categories of possible applications motivating the usage of these special curves in the context of computational simulations. Two variants for the construction of (discrete iterations of) the curves are explained in detail such that the reader is in the position to use space-filling curves for a tangible tasks like ordering Cartesian mesh cells. Here, strong connections to spacial discretisation (cf. Chap. 2) and its efficient implementation are provided.

Part IV is devoted to a more in depth study of the theory and simulation of random (ordinary) differential equations. It analyses the theory of linear random differential equations. Numerical schemes for (non-linear) random differential equations, like the the averaged Euler and Heun method are discussed. Stability of the null-solution is considered and Lyapunov-type meth- 
ods are applied to the various concepts of stochastic stability. Finally, the recent theory of random dynamical systems and its impacts on the study of random (ordinary) differential equations is presented.

Chapter 12 treats linear inhomogeneous ordinary random differential equations of the type $\dot{X}_{t}=A(t) X_{t}+Z_{t}$ where the randomness is located just in the inhomogeneous driving process $Z_{t}$. These types of equations can be analysed in ways analogous to their deterministic counterparts already exhibiting a wealth of interesting phenomena. Of importance are the stochastic characteristics of the solutions process as well as periodic and stationary solution types. In particular, we give first stability conditions with respect to which solutions converge towards periodic or stationary ones.

Chapter 13 extends this body of knowledge on linear random ordinary differential equations by also allowing stochastic effects in the coefficients. We give the general solution formulas for these types of equations together with equivalence result for path-wise and mean-square solutions. Moreover, on the one hand, we analyse the asymptotic properties of path-wise solutions focusing on (exponential) decay towards the nullsolution as well as on upper bounds for path-wise solutions. On the other hand, we also study the properties of the moments of path-wise solutions with respect to the (exponential) decay as well as the existence of asymptotically $\theta$-periodic solutions. As an excursion, the general solution formula of linear non-commutative path-wise continuous noise systems is constructed.

Chapter 14 discusses all relevant aspects for simulation of path-wise RODE problems. We present lower-order explicit RODE schemes (Euler and Heun) as well as higher-order K-RODE Taylor schemes. Detailed information on the corresponding MATLAB implementation for the wireframe model are given and numerical results show the validity of the approach.

Chapter 15 studies the various notions of stability of the null solution of a random (ordinary) differential equation with a focus on path-wise equistability, $h$-, $\mathbb{P}$-, and $W$-stability. In particular, the relations/ implications and inter-connections between these concepts are discussed and the results of Chap. 13 on the path-wise stability of linear random differential equations with stochastic coefficients are re-framed in the context of these concepts. Moreover, we extend the deterministic Lyapunov method to random differential equations. Based on suitable Lyapunov-functions, necessary conditions for $h$-stability and path-wise equi-stability are given. Finally, the stability of deterministic systems 
subject to different classes of continuously acting random perturbations is analysed.

Chapter 16 provides a glimpse into the very recent theory of random dynamical systems. We give the fundamental definitions of metric, measurable and random dynamical systems together with some illustrative examples. Moreover, we study the notions of forward and backwards stability and their implications.

Part $\mathrm{V}$ gives the problem set of the workshop associated to the course we gave in the summer term 2012 together with some key results and lessons learnt from this experiment in higher education.

Chapter 17 focuses on the didactic aspects of the workshop. We discuss the integration of workshop as a central part of the complete course. Details on the design of the workshop are presented covering in particular the concept of a virtual software company, the choice of the environment, and the team role descriptions.

Chapter 18 contains the project specification used in the workshop. We present a selection of example results which our students produced at the end of the project. Finally, we summarise the lessons learnt-both from the point of view of the participants and the supervisors-providing interesting hints for future or similar projects. 